\title{
Tensión, deformación y fisurabilidad
}

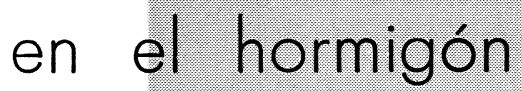

J. M. TOBio, Dr. en Química Industrial

I.E.T.C.C.C.

\section{SINOPSIS}

En este trabajo se insiste en el concepto del "límite de fisuración intensiva", del hormigón y en los criterios no destructivos para determinarlo.

Por otra parte, se hace una breve revisión sobre las teorías que se refieren a la macroestructura de las pastas y su influencia en el mecanismo de rotura, de acuerdo con las hipótesis más generalmente admitidas.

\section{CONSIDERACIONES SOBRE LA MACROESTRUCTURA DE LAS PASTAS DE CEMENTO Y HORMIGON}

La estructura del hormigón puede ser considerada como una red espacial compuesta de granos resistentes (el árido) sumergidos en un magma de pasta de cemento endurecida, comprendiendo, además, huecos, poros, microporos, canales capilares, intersticios llenos de aire, vapores (de agua, por ejemplo), gases, agua libre y combinada físicamente, interfases árido-pasta y árido-árido, etc.

La pasta de cemento presenta una estructura similar a la del hormigón, exceptuando el árido, que aquí es reemplazado por granos de cemento sin hidratar $\multimap$ en curso de hidratación - así como por concreciones cristalinas, rodeado todo ello por el gel de hidrosilicatos en proceso continuo de endurecimiento.

Desde el punto de vista estático, la estructura del hormigón puede imaginarse como un sistema espacial en forma de red de hiperestaticidad elevada. Bajo la acción de solicitaciones diversas, este sistema, formado por elementos de diferente rigidez unidos entre sí, se carga y sufre deformaciones compatibles con las uniones o enlaces existentes en la red estructural.

Las tensiones y deformaciones de larga duración se redistribuyen continuamente como consecuencia de la aparición de deformaciones plásticas que caracterizan el comportamiento de los geles, así como bajo el efecto de las rupturas locales aparecidas por la modificación de enlaces entre los elementos, que dan lugar a la aparición y desarrollo de las microfisuras. 
La característica más destacable de este tipo de estructuras es la aparición de concentraciones de tensiones - tanto las provocadas por una solicitación externa como por las internas propias del sistema- cuyos valores pueden alcanzar a varias veces el valor medio de las tensiones.

Todo ello no es más que el resultado de la heterogeneidad del hormigón. Se puede demostrar, tanto teórica como experimentalmente, que la influencia de los granos de árido por relación a los huecos interviene como efecto decisivo en el comportamiento elástico del hormigón.

Parece indudable que las causas que preparan la aparición de microfisuras - y, por tanto, la rotura del hormigón - son los huecos, los poros, las zonas débiles en la proximidad de los áridos y otras imperfecciones del conjunto.

\section{MECANISMO DE LA ROTURA}

Cuando se somete el hormigón a compresión tiene lugar una degradación progresiva de la estructura, la aparición de fisuras orientadas en la dirección de la carga y, finalmente, la rotura del elemento.

En el caso de las cargas de tracción, la rotura es esencialmente diferente de la que se produce por compresión: La pérdida de la capacidad portante del hormigón sometido a tensiones de tracción tiene una característica autoacelerada, la aparición de tensiones locales provoca el nacimiento de nuevos defectos y no puede hablarse de fenómenos de adaptación. Nos referimos, por supuesto, al hormigón en masa.

La rotura por tracción se produce en una forma cuasi-frágil propia de los materiales quebradizos.

La forma, situación y reparto estadístico de los huecos poseerá, por tanto, un efecto decisivo en el comportamiento del hormigón bajo carga, En casos especiales, tales huecos pueden conducir incluso a la disminución del crecimiento del grado de concentración de tensiones.

La pérdida de la capacidad portante del hormigón, tanto en compresión como en tracción, puede ser explicada, pues, mediante los fenómenos que preceden a la rotura y que aparecen en la microestructura mucho tiempo antes de que se produzca la rotura propiamente dicha. La duración, magnitud y velocidad de aplicación de la carga juegan también un papel importante en este proceso.

En los elementos comprimidos, los cambios de forma de los huecos existentes tienen por efecto una reducción del grado de concentración de tensiones de compresión.

Las cosas se complican aún más cuando existen "obstáculos" en la masa del hormigón, como es el caso del hormigón armado. Pero el estudio de todo esto nos llevaría demasiado lejos en estas consideraciones.

\section{DEFORMACIONES, TENSION Y FISURACION}

Operando con cargas de corta duración, la rotura del hormigón se produce cuando se alcanza la tensión crítica. El valor de ésta señala un estado físico tal del elemento de hor- 
migón, durante el cual un cambio de volumen específico del mismo provocado por una carga, alcanza su valor máximo mientras que el volumen llega a ser el mínimo.

Dicho cambio de volumen viene definido por:

$$
\Delta \theta=\varepsilon_{1}-2 \varepsilon_{\mathrm{t}},
$$

siendo $\varepsilon_{1}$ la deformación longitudinal y $\varepsilon_{t}$ la transversal.

Otra forma de definir este límite es por medio del coeficiente de Poisson:

$$
\nu=\frac{\Delta \varepsilon_{\mathrm{t}}}{\Delta \varepsilon_{1}}
$$

que alcanza el valor máximo de 0,5 cuando se llega al límite intensivo de fisuración $\mathrm{R}_{\text {fis }}$.

Este límite puede ser determinado, según las técnicas de medida más recientes, mediante un criterio que tenga en cuenta la velocidad de propagación de una onda elástica ultrasónica, así como también por las variaciones de las deformaciones longitudinal y transversal.

La experiencia ha mostrado que existe una correlación entre la velocidad ultrasónica, la tensión y las deformaciones, incluso dentro del campo no-lineal, cuando se produce la microfisuración.

\section{LOS CRITERIOS DE DETERMINACION DEL LIMITE DE FISURACION INTENSIVA}

Utilizando ambos sistemas de medida, la velocidad de la onda ultrasónica y la extensometría, se puede definir el límite de fisuración intensiva. Para piezas sometidas a carga centrada de corta duración, el siguiente criterio:

$$
\Delta \mathrm{V}_{\mathrm{i}}^{\mathrm{fis}}=\frac{\mathrm{V}_{\mathrm{i}}-\mathrm{V}_{\mathrm{i}+1}}{\mathrm{~V}_{\mathrm{o}}}>0,02,
$$

donde $\mathrm{V}_{0}$ es la velocidad de los impulsos ultrasónicos para carga cero y $\mathrm{V}_{\mathrm{i}}$ y $\mathrm{V}_{\mathrm{i}+1}$ son las velocidades para dos escalones de carga sucesivos, es suficientemente exacto y las medidas muy fáciles de llevar a cabo.

En efecto, basta observar que la velocidad de los impulsos ultrasónicos cae más del $2 \%$ para dos escalones sucesivos de carga que difieran entre sí por lo menos un $5 \%$ de la carga límite.

Si se trata de una pieza prismática, como puede ser una probeta, deben medirse al menos tres niveles de la misma para tener la seguridad de que todo el posible campo de aparición de las microfisuras en el elemento queda cubierto. Las velocidades para cada uno de estos niveles las denominaremos $\mathrm{V}_{\mathrm{s}}, \mathrm{V}_{\mathrm{m}} \mathrm{y} \mathrm{V}_{\mathrm{i}}$. 


\begin{tabular}{|c|c|c|}
\hline 9 & $\therefore$ & 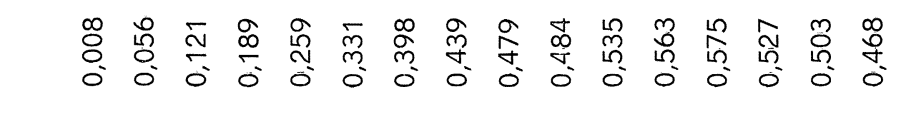 \\
\hline 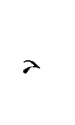 & & 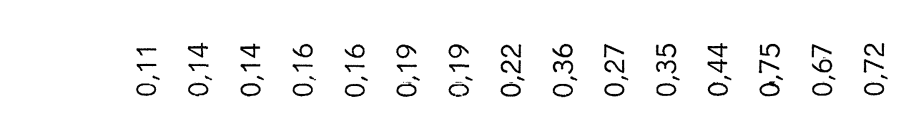 \\
\hline$\stackrel{\omega}{\triangleleft}$ & $\therefore$ & 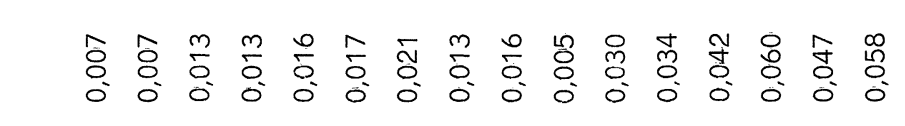 \\
\hline$\stackrel{\omega}{4}$ & $\therefore$ & 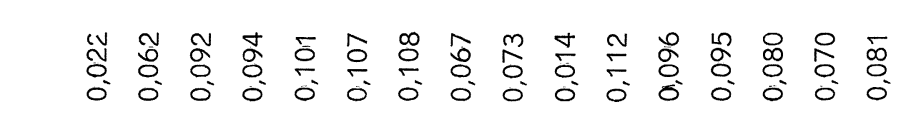 \\
\hline$\omega$ & $\therefore$ & 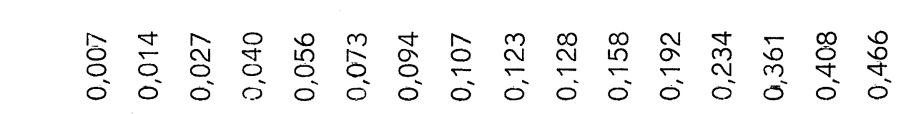 \\
\hline$\omega$ & $\therefore$ & 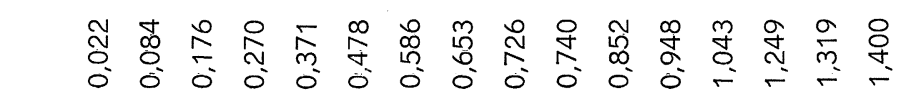 \\
\hline$\stackrel{5}{4}$ & $\therefore$ & 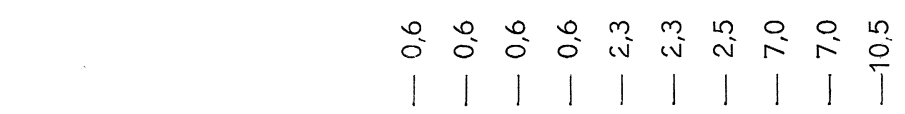 \\
\hline$>$ & $\widehat{\underline{s}}$ & 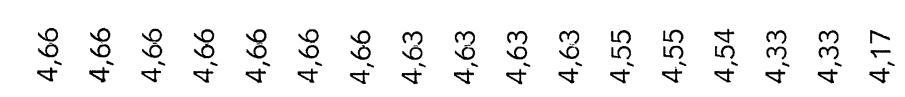 \\
\hline$\stackrel{8}{3}$ & $\therefore$ & 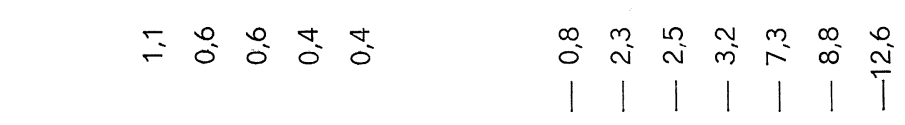 \\
\hline$>$ & $\underline{\underline{\underline{s}}}$ & 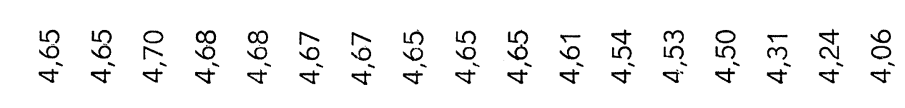 \\
\hline 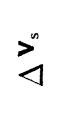 & $\therefore$ & 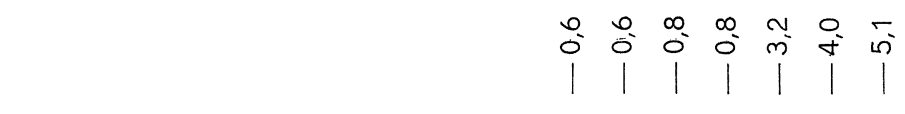 \\
\hline$>$ & $\underline{\underline{\underline{n}}}$ & 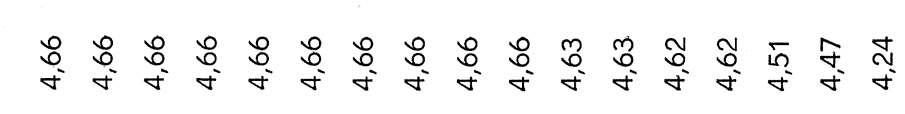 \\
\hline$\therefore$ & $\therefore$ & 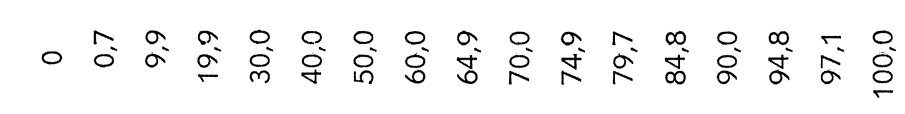 \\
\hline 。 & 索 & 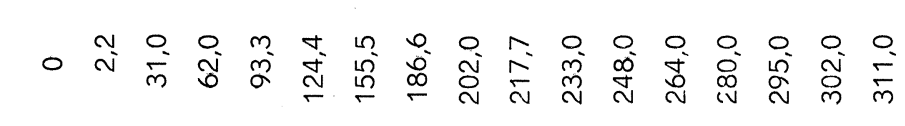 \\
\hline
\end{tabular}


La tabla I resume los datos obtenidos en una experiencia de rotura en compresión de probetas prismáticas de hormigón (resistencia prevista del mismo $300 \mathrm{kp} / \mathrm{cm}^{2}$ ), sometidas a carga centrada de corta duración.

En dicha tabla se indican los escalones de carga, el porcentaje de la carga de rotura, las velocidades ultrasónicas según tres trayectorias diferentes, los incrementos de estos valores, las deformaciones longitudinal y transversal, sus incrementos, el coeficiente de Poisson y los incrementos de volumen.

Las velocidades se pueden determinar fácilmente con un aparato ultrasónico normal o digital, y las deformaciones por medio de strain-gauges (bandas extensométricas) y el puente de medida correspondiente.

Puede observarse en la tabla que el límite de la fisuración intensiva se alcanza para la carga $\sigma_{\mathrm{fis}}=280 \mathrm{kp} / \mathrm{cm}^{2}$, puesto que:

$$
\frac{\mathrm{V}_{\mathrm{i}}-\mathrm{V}_{\mathrm{i}+1}}{\mathrm{~V}_{0}}=\frac{4,54-4,33}{4,66}=0,0451>0,02
$$

El valor de esta carga crítica de fisuración viene a ser un 0,9 de la carga de rotura.

Según el criterio del coeficiente de Poisson antes señalado, $\nu=0,5$ para $\sigma_{\mathrm{fis}}=290 \mathrm{kp} /$ $/ \mathrm{cm}^{2}$, o sea, un 0,93 de la carga de rotura.

Si aplicamos el criterio de las deformaciones o del límite de la tensión crítica, hallamos para $\sigma_{\text {fis }}$ un valor comprendido entre 280 y $295 \mathrm{kp} / \mathrm{cm}^{2}$, es decir, entre $0,9 \sigma$ y $0,948 \sigma$.

Se trata, como puede verse, de valores muy concordantes que muestran que, a $280 \mathrm{kp} / \mathrm{cm}^{2}$, el hormigón ha entrado en su fase de deterioro por microfisuración intensiva, aun cuando no se haya alcanzado la carga de rotura en prensa.

Para dicha solicitación de $0,9 \sigma$ puede decirse que el hormigón está irreversiblemente dañado, aun cuando no se haya producido la rotura.

Existe, en virtud de estos resultados, una relación entre tensión, deformación y velocidad de impulsos ultrasónicos en el hormigón, dentro del campo no-lineal de cargas de compresión, que viene dada por una ecuación parabólica de segundo grado con tres constantes.

\section{BIBLIOGRAFIA}

(1) BERG, O. J.: "Wissenschaftliche Zeitschrift der TU Dresden", 17, H.6. (1968).

(2) Brandtzaeg, A.: "Norges Teknische Hoiskole" (1935).

(3) L'Hermite, R.: “Annales de l'ITBTP”, 9 (1957).

(4) Jones, R.: "British Journal of Applied Physics", vol. 3, núm. 7 (1952).

(5) GalaN, A.: "Rapport RILEM”, vol. II, pág. 172, (1974).

(6) FACAOARU, I.: “RILEM Working Group for NDT. Aachen Meeting” (1968).

(7) Mrhul, A.: “Rapport RILEM”, vol. III, pág. 196 (1974). 